\title{
The phase diagram in the vector meson extended linear sigma model*
}

\author{
Gy. Wolf And P. Kovács
}

Wigner Research Centre for Physics, Hungarian Academy of Sciences, H-1525

Budapest, POB 49, Hungary

\begin{abstract}
We investigate the chiral phase transition of the strongly interacting matter at nonzero temperature and baryon chemical potential $\mu_{B}$ within an extended $(2+1)$ flavor Polyakov constituent quark-meson model which incorporates the effect of the vector and axial vector mesons. The parameters of the model are determined by comparing masses and tree-level decay widths with experimental values. We examine the restoration of the chiral symmetry by monitoring the temperature evolution of condensates. We study the $\mathrm{T}-\rho_{B}$ phase diagram of the model and find that a critical end point exists, although at very low density.
\end{abstract}

PACS numbers: 12.39.Fe, 11.30.Rd, 11.30.Qc, 14.40.Be

\section{Introduction}

We investigate the thermodynamics of the strongly interacting matter at high temperature and/or density with the $(2+1)$ flavor Polyakov quark meson model in which, beyond the vector and axial vector mesons included alongside the scalar and pseudoscalar ones. We take into account, as fermionic degrees of freedom, the constituent quarks propagating on a constant gluon background in the temporal direction, which naturally leads in a mean-field treatment to the appearance of the Polyakov loop. We incorporate the vacuum fluctuations of the fermions in the grand potential. We compare our results at $\mu_{B}=0$ with lattice data and then move to finite $\mu_{B}$. The details of the model can be found in [1, 2].

\section{Formulation of the Model}

We work with the Lagrangian used in [2]. Compared to the usual chiral Lagrangian, we introduce additional kinetic and Yukawa coupling terms for

\footnotetext{
* CPOD 2016, Wroclaw
} 
the constituent quarks $\Psi=\left(q_{u}, q_{d}, q_{s}\right)^{T}$. Another important modification is the presence of the gluon field in the covariant derivative of the quark field. In the mean-field approximation, this will give rise in the grand potential to the appearance of the Polyakov loop, which mimics some properties of the quark confinement. We use the logarithmic Polyakov loop potential [3]. This parametrization does not include the backreaction of the dynamical quarks on the gauge sector and therefore the influence of the quarks on the deconfinement phase transition. In the present study we shall use the improved Polyakov loop potential proposed in [4].

There are altogether 16 unknown parameters, 15 parameters found in the Lagrangian and the renormalization scale. In the parametrization procedure we use alongside 29 vacuum quantities, and also the pseudocritical temperature $T_{c}$ at $\mu_{B}=0$ [2]. The value of the masses and decay constants are compared with the corresponding experimental value taken from the PDG [5]. The $T_{c}$ pseudocritical temperature, which should be around $150 \mathrm{MeV}$, can reach very high values $(\geq 350 \mathrm{MeV})$ in case of some solutions. Thus we chose to include the physical value of $T_{c}$ in the parametrization with a $10 \%$ error to throw away solutions with high $T_{c}$, and unrealistic thermodynamics.

\subsection{The Grand Potential}

To study the thermodynamics of a symmetric quark matter $\left(\mu_{u}=\mu_{d}=\right.$ $\left.\mu_{s} \equiv \mu_{q}=\mu_{B} / 3\right)$, we shall use the grand potential $\Omega\left(T, \mu_{q}\right)$ obtained from the partition function of a three-dimensional spatially uniform system of volume $V$ in thermal equilibrium at temperature $T=1 / \beta$. Since the Polyakov loop is treated at mean-field level, there is no integration over the gluons and in this case the Polyakov loop potential is simply added to the grand potential.

In the present case the vacuum and thermal fluctuations for the fermions are taken into account, while the mesonic vacuum fluctuations are neglected and the effects of the lightest mesonic thermal fluctuations $\left(\pi, K, f_{0}^{L}\right)$ are included only in the pressure and the thermodynamical quantities derived from it. In this approximation the grand potential reads

$$
\Omega_{\mathrm{H}}\left(T, \mu_{q}\right)=U(\langle M\rangle)+U(\langle\Phi\rangle,\langle\bar{\Phi}\rangle)+\Omega_{\bar{q} q}^{(0)}\left(T, \mu_{q}\right),
$$

where $U(\langle M\rangle)$ is the tree-level meson potential, $U(\langle\Phi\rangle,\langle\bar{\Phi}\rangle)$ is the Polyakov loop potential and $\Omega_{\bar{q} q}^{(0)}$ is the contribution of the fermions. 


\subsection{The curvature meson masses}

The squared masses of the scalar and pseudoscalar mesons are calculated from the elements of the corresponding curvature matrix:

$$
m_{i, a b}^{2}=\left.\frac{\partial^{2} \Omega\left(T, \mu_{q}\right)}{\partial \varphi_{i, a} \partial \varphi_{i, b}}\right|_{\min }=\mathrm{m}_{i, a b}^{2}+\Delta m_{i, a b}^{2}+\delta m_{i, a b}^{2}
$$

where the three terms on the right-hand side are as follows: $\mathrm{m}_{i, a b}^{2}$ is the tree-level mass matrix, and $\Delta m_{i, a b}^{2}$ and $\delta m_{i, a b}^{2}$ are the contributions of the fermionic vacuum and thermal fluctuations, respectively.

The field equations, which determine the dependence on $T$ and $\mu_{B}=3 \mu_{q}$ of the chiral condensates $\phi_{N}$ and $\phi_{S}$ and the Polyakov loop variables $\Phi$ and $\bar{\Phi}$, are obtained by extremizing the grand potential,

$$
\frac{\partial \Omega_{\mathrm{H}}}{\partial \phi_{N}}=\frac{\partial \Omega_{\mathrm{H}}}{\partial \phi_{S}}=\frac{\partial \Omega_{\mathrm{H}}}{\partial \Phi}=\frac{\partial \Omega_{\mathrm{H}}}{\partial \bar{\Phi}}=0 .
$$

To calculate various thermodynamical quantities we add the contribution of a meson $b \in\left\{\pi, K, f_{0}^{L}\right\}$ to the pressure with the formula

$$
\Delta p_{b}(T)=-n_{b} T \int \frac{d^{3} p}{(2 \pi)^{3}} \ln \left(1-e^{-\beta E_{b}(p)}\right),
$$

where $E_{b}(p)=\sqrt{p^{2}+m_{b}^{2}}$, with $m_{b}$ being the meson mass, and $n_{b}$ is the meson multiplicity $\left(n_{\pi}=3, n_{K}=4\right.$, and $\left.n_{f_{0}^{L}}=1\right)$.

\section{Results}

On the left hand side of Fig. 1 we study at $\mu_{B}=0$ the temperature variation of the nonstrange and strange chiral condensates and Polyakov loop expectation value. We see that the chiral condensates stay close to their vacuum values up to some quite high value of the temperature of order $100 \mathrm{MeV}$.

On the right hand side of Fig. 1 we see that the constituent quarks and the Polyakov loop potential give the major part of the contribution to the pressure around and beyond $T_{c}$ and that at small temperature the pressure is pion dominated.

Some thermodynamical quantities derived from the pressure, like the scale interaction measure $\Delta$, the square of the speed of sound $c_{s}^{2}$, and the equation of state parameter $p / \epsilon$ (pressure over energy density), are shown in [2] to be described very well by the model. 

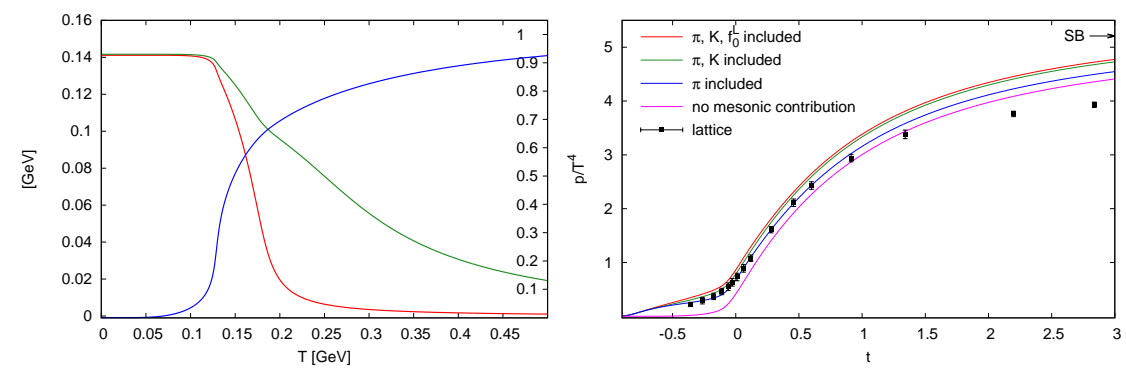

Fig. 1. At $\mu_{B}=0$ temperature dependence of condensates from the improved Polyakov loop potential (left) and the pressure (right) as function of reduced temperature $\left(T / T_{c}-1\right)$. Lattice data are taken from [6]. Here $T_{c}^{\text {glue }}=182 \mathrm{MeV}$.

\section{1. $\mu_{B}-T$ phase diagram and the critical endpoint}

We turn now to the study of the chiral phase transition at finite quark chemical potentials: $\mu_{u}=\mu_{d}=\mu_{s}=\mu_{q}=1 / 3 \mu_{B}$. The lattice result of the curvature at $\mu_{B}=0$ is very well reproduced [2]. With the best set of parameters, the location of the CEP in our model is given by $\left(\mu_{B}^{\mathrm{CEP}}, T_{c}^{\mathrm{CEP}}\right)=$ $(885,52.7) \mathrm{MeV}$ when the improved Polyakov loop potential is used with $T_{c}^{\text {glue }}=210 \mathrm{MeV}$. The phase diagram is similar to that obtained in [7], with comparable values of the CEP's coordinates. The large value of $\mu_{B}^{\mathrm{CEP}}$ we obtained is typical of a linear sigma model without (axial)vector mesons in the case when the vacuum fluctuation of the fermions is included. See, e.g., 8 where the value $\left(\mu_{B}^{\mathrm{CEP}}, T_{c}^{\mathrm{CEP}}\right)=(849,81) \mathrm{MeV}$ was reported. Without the inclusion of the fermionic vacuum fluctuations, as is the case of Refs. [9, 10], the value of $\mu_{B}^{\mathrm{CEP}}$ is smaller and $T_{c}^{\mathrm{CEP}}$ higher, compared to the case when they are properly taken into account.

On Fig. 2 we show the phase diagram on the $T, \rho_{B}$ plane. The dashed line shows the first order transition line whereas the solid curve is where the transition is cross over. The critical point is at rather low density, note, however, it is not the usual $\mu_{u}=\mu_{d}=\mu_{q}=1 / 3 \mu_{B}, \mu_{s}=0$ case.

\section{Conclusions}

We have studied at finite temperature and baryonic densities the thermodynamical properties of the Polyakov loop extended quark meson model containing also vector and axial vector mesons. These latter ingredients manifest themselves in a nontrivial way in the vacuum parametrization of the model through their tree-level masses and decay widths. The $\chi^{2}$ minimization procedure was applied to fix the parameters. We have studied 


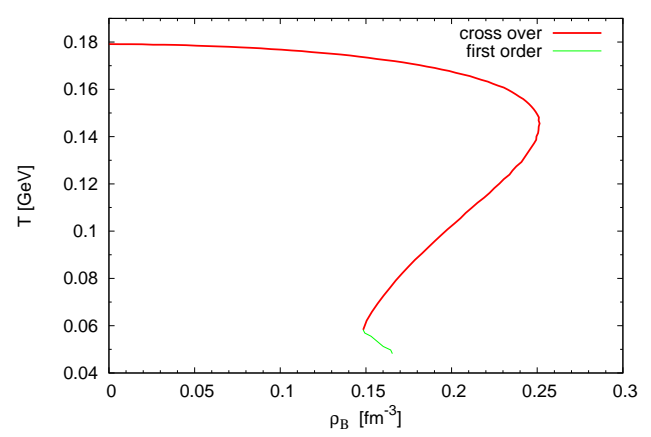

Fig. 2. The phase diagram obtained by using the improved Polyakov loop potential. The dashed line shows the first order phase transition usinf Maxwell construction.

the thermodynamics of the model, by using an improved Polyakov loop potential, and obtained fairly good agreement with lattice data. We found that a CEP of the crossover transition line exists in the $T-\rho_{B}$ phase diagram at rather low values of $\rho_{B}$.

\section{Acknowledgments}

The authors were supported by the Hungarian Research Fund (OTKA) under Contract No. K109462 and by the HIC for FAIR Guest Funds of the Goethe University Frankfurt.

\section{REFERENCES}

[1] D. Parganlija, P. Kovács, Gy. Wolf, F. Giacosa and D. H. Rischke, Phys. Rev. D 87, 014011 (2013).

[2] P. Kovács, Zs. Szép and Gy. Wolf, Phys. Rev. D 93, 114014 (2016).

[3] K. Fukushima, Phys. Lett. B 591, 277 (2004).

[4] L. M. Haas, R. Stiele, J. Braun, J. M. Pawlowski and J. Schaffner-Bielich, Phys. Rev. D 87, 076004 (2013).

[5] J. Beringer et al. [PDG], Phys. Rev. D 86, 010001 (2012).

[6] S. Borsányi, G. Endrődi, Z. Fodor, A. Jakovác, S. D. Katz, S. Krieg, C. Ratti and K. K. Szabó, JHEP 1011, 077 (2010).

[7] R. Stiele and J. Schaffner-Bielich, Phys. Rev. D 93, 094014 (2016).

[8] S. Chatterjee and K. A. Mohan, Phys. Rev. D 85, 074018 (2012).

[9] B. J. Schaefer and M. Wagner, Phys. Rev. D 79, 014018 (2009).

[10] H. Mao, J. Jin and M. Huang, J. Phys. G 37, 035001 (2010). 